\title{
Efficacies of minimally invasive puncture and small bone window craniotomy for hypertensive intracerebral hemorrhage, evaluation of motor-evoked potentials and comparison of postoperative rehemorrhage between the two methods
}

\author{
LEI LUAN, MAOLEI LI, HANG SUI, GUOLIANG LI and WENYONG PAN \\ Department of Neurosurgery, Chengyang People's Hospital, Qingdao, Shandong 266109, P.R. China
}

Received April 27, 2018; Accepted November 5, 2018

DOI: $10.3892 /$ etm.2018.7094

\begin{abstract}
Application value of the minimally invasive puncture and small bone window craniotomy in hypertensive intracerebral hemorrhage was investigated to explore the effects of the above treatment methods on motor-evoked potentials (MEPs) and postoperative rehemorrhage. Patients with hypertensive intracerebral hemorrhage who were admitted to Chengyang People's Hospital from March 2016 to December 2017 were selected and randomly divided into the minimally invasive group $(n=40)$ and the craniotomy group $(n=40)$. The minimally invasive group was treated with minimally invasive puncture and drainage for hematomas, while the craniotomy group received small bone window craniotomy for evacuation of hematomas. The clinical efficacy was compared between the two groups. At 28 days after operation, the Chinese scale of clinical neurological deficit of stroke patients (CSS) score in the minimally invasive group was lower than that in the craniotomy group $(\mathrm{p}<0.05)$. At 28 days after operation, the $\mathrm{S}-100 \beta$ level in the minimally invasive group was lower than that in the craniotomy group $(\mathrm{p}<0.05)$. At 1 week after operation, 35 patients in the minimally invasive group were able to elicit MEP waveforms, and only 7 patients in the craniotomy group were able to elicit positive waveforms. At 2 weeks after operation, 40 patients in the minimally invasive group and 20 patients in the craniotomy group could elicit MEP waveforms, and the incubation period, central motor conduction time and amplitude in the former were significantly better than those in the latter $(\mathrm{p}<0.05)$. The operation time and length of hospital stay were
\end{abstract}

Correspondence to: Dr Lei Luan, Department of Neurosurgery, Chengyang People's Hospital, 600 Changcheng Road, Qingdao, Shandong 266109, P.R. China

E-mail: cyah338@163.com

Key words: minimally invasive puncture, small bone window craniotomy, hypertensive intracerebral hemorrhage, motor-evoked potential, complication shorter with more total expenses of hospitalization in the minimally invasive group compared to those in the craniotomy group $(\mathrm{p}<0.05)$. Compared with small bone window craniotomy, minimally invasive puncture can reduce serum $\mathrm{S}-100 \beta$ level. Its advantages are obvious, so it is worthy of promotion and application.

\section{Introduction}

Hypertensive intracerebral hemorrhage is a non-traumatic brain parenchymal hemorrhage disease with the highest incidence rate in the cold season. Since the cold climate induces contraction of blood vessels in the human body, blood pressure fluctuates greatly, which easily causes blood vessel rupture resulting in hemorrhage (1). However, the pathological mechanism of hypertensive intracerebral hemorrhage is more complicated, which is related not only to the cold climate factors, but also to the formation of intracranial microaneurysms, intracranial arterial wall degeneration and necrosis and cerebrovascular amyloidosis (2). The disability and mortality rates of hypertensive intracerebral hemorrhage are high, so the improvement of clinical efficacy of these methods has become a research hotspot in recent years (3). At present, minimally invasive puncture and small bone window craniotomy are the main treatment methods for hypertensive intracerebral hemorrhage, but their advantages and disadvantages are not yet clear (4). In particular, there are few studies and reports on the effects of the two surgical methods on postoperative motor-evoked potentials (MEPs) of patients (5). This study compared the efficacy between minimally invasive puncture and small bone window craniotomy in the treatment of hypertensive intracerebral hemorrhage, and explored the effects of the above treatment methods on MEPs and postoperative rehemorrhage, so as to provide a basis for treating hypertensive intracerebral hemorrhage.

\section{Patients and methods}

General data. A total of 80 patients with hypertensive intracerebral hemorrhage admitted and treated in Chengyang People's Hospital (Qingdao, China) from March 2016 to December 2017 were selected for the study. Inclusion criteria: i) patients 
Table I. Comparison of general data between the two groups of patients.

\begin{tabular}{|c|c|c|c|c|}
\hline Variables & $\begin{array}{l}\text { Minimally invasive } \\
\text { group }(\mathrm{n}=40)\end{array}$ & $\begin{array}{l}\text { Craniotomy } \\
\text { group }(\mathrm{n}=40)\end{array}$ & $t / \chi^{2}$ & P-value \\
\hline Age (years) & $56.35 \pm 3.21$ & $57.41 \pm 4.08$ & 0.682 & 0.095 \\
\hline \multicolumn{5}{|l|}{$\operatorname{Sex}(n)$} \\
\hline Male & 21 & 22 & \multirow[t]{2}{*}{1.074} & \multirow[t]{2}{*}{0.083} \\
\hline Female & 19 & 18 & & \\
\hline Body mass index $\left(\mathrm{kg} / \mathrm{m}^{2}\right)$ & $25.13 \pm 1.73$ & $25.15 \pm 2.41$ & 0.521 & 0.166 \\
\hline Volume of hematoma $(\mathrm{ml})$ & $42.56 \pm 4.31$ & $43.06 \pm 5.08$ & 0.785 & 0.103 \\
\hline Glasgow Coma Scale score (points) & $10.11 \pm 1.54$ & $10.24 \pm 1.69$ & 1.092 & 0.065 \\
\hline \multicolumn{5}{|l|}{ Operation timing (n) } \\
\hline At $12 \mathrm{~h}$ after onset & 20 & 21 & \multirow[t]{3}{*}{1.336} & \multirow[t]{3}{*}{0.072} \\
\hline At $12-24 \mathrm{~h}$ after onset & 16 & 15 & & \\
\hline At $24-72 \mathrm{~h}$ after onset & 4 & 4 & & \\
\hline
\end{tabular}

definitely diagnosed with hypertensive intracerebral hemorrhage (6); and ii) patients undergoing surgical treatment within $72 \mathrm{~h}$ of onset with postoperative follow-up period $\geq 3$ months. Exclusion criteria: i) patients with cerebral hemorrhage caused by trauma or other non-hypertensive factors; ii) patients who were in critical condition with no time for surgical treatment; iii) patients with diseases in the liver, kidney or other important organs; iv) psychopaths; or v) women in the gestation period. These 80 patients were randomly divided into the minimally invasive group $(n=40)$ and the craniotomy group $(n=40)$. Comparisons of general data between the two groups of patients are shown in Table I.

This study was approved by the Ethics Committee of Chengyang People's Hospital. Signed informed consents were obtained from the patients or the guardians.

Treatment methods. The minimally invasive group was treated with minimally invasive puncture and drainage for hematoma: hematoma lesions were found by computed tomography (CT) before operation, minimally invasive puncture points were marked on the patient's scalp, and the direction and depth of minimally invasive needle insertion were scientifically evaluated. The patients were locally hypodermically injected with $1.0 \%$ lidocaine according to a method described in a previous study (7). Electric drill was applied to gradually insert the puncture needle into the patient's intracranial hematoma lesions. After the needle was pulled out, the hematoma aspiration was performed. When the hematoma volume reached $\sim 50 \%$, the aspiration was stopped.

The craniotomy group underwent small bone marrow craniotomy: the patients were generally anesthetized using the procedure and were examined via $\mathrm{CT}$ before operation in order to view the lesions that were away from important blood vessels and functional areas of the brain. Generally, bone windows with a diameter of $\leq 4 \mathrm{~cm}$ were selected at the closest point near the hematoma lesions. After the patient's endocranium was incised, the hematomas were aspirated with aspiration volume of 60-92\%. After hemostasis, a drainage tube was placed on the patient's hematoma cavity.
Urokinases were injected into the patient's hematoma cavity postoperatively, and if there were no symptoms of intracranial hypertension, open drainage was conducted $4 \mathrm{~h}$ after injection once a day. The patients were subjected to regular cranium CT re-examination after operation. If the hematoma volume was $<10 \mathrm{ml}$, the drainage tube could be removed. Both groups of patients were treated with routine treatments such as anti-infective and neurotrophic treatments after operation.

\section{Observation indexes}

Operation-related indexes. The operation time, drainage tube removal time, length of hospital stay and the total expenses of hospitalization of patients were recorded and compared.

Neurological function. Before operation and at 28 days after operation, the Chinese scale of clinical neurological deficit of stroke patients (CSS) (8) was adopted to evaluate the neurological deficit scores of the two groups of patients.

Ability to daily living activities. Barthel index (9) was employed to assess the ability to daily living activities of the two groups of patients at 3 months after operation.

MEPs. Neurotic electrophysiology was utilized to detect MEPs in the two groups of patients (10).

Serum $S-100 \beta$. Changes in the level of serum S-100 $\beta$ in the two groups of patients before operation and at $1,3,7$, 14, 21 and 28 days after operation were determined via the enzyme-linked immunosorbent assay (ELISA).

Complications. After 3 months of follow-up, postoperative complications such as postoperative rehemorrhage or new intracranial hemorrhage, multiple organ failure and pulmonary embolism were observed and recorded.

Statistical analysis. Statistical Product and Service Solutions (SPSS) 21.0 software was used for data analysis. Enumeration data were also expressed as percentage (\%). Chi-square test was used for intergroup comparisons. Measurement data were expressed as mean \pm standard deviation (SD), and the t-test was applied for intragroup and intragroup comparisons. $\mathrm{P}<0.05$ was considered to indicate a statistically significant difference. 
Table II. Comparison of postoperative complications between the two groups [n (\%)].

\begin{tabular}{|c|c|c|c|c|c|c|}
\hline Group & $\mathrm{n}$ & Stress ulcer & $\begin{array}{l}\text { Rehemorrhage or } \\
\text { new intracranial } \\
\text { hemorrhage }\end{array}$ & $\begin{array}{c}\text { Multiple } \\
\text { organ failure }\end{array}$ & $\begin{array}{l}\text { Pulmonary } \\
\text { embolism }\end{array}$ & Mortality \\
\hline Minimally invasive group & 40 & $3(7.50)$ & $3(7.50)$ & $2(5.00)$ & $1(2.50)$ & $6(15.00)$ \\
\hline Craniotomy group & 40 & $2(5.00)$ & $3(7.50)$ & $1(2.50)$ & $1(2.50)$ & $5(12.50)$ \\
\hline$\chi^{2}$ & & 0.962 & 0.856 & 1.008 & 1.145 & 0.697 \\
\hline P-value & & 0.154 & 0.097 & 0.133 & 0.098 & 0.086 \\
\hline
\end{tabular}

Table III. Comparison of the CSS score between the two groups (mean \pm SD, points).

\begin{tabular}{|c|c|c|c|c|c|}
\hline Group & $\begin{array}{c}\text { No. of } \\
\text { survived cases }\end{array}$ & Before operation & $\begin{array}{l}\text { At } 28 \text { days } \\
\text { after operation }\end{array}$ & $\mathrm{t}$ & P-value \\
\hline Minimally invasion group & 34 & $40.96 \pm 6.15$ & $20.04 \pm 3.51$ & 21.095 & $<0.001$ \\
\hline Craniotomy group & 35 & $40.87 \pm 5.32$ & $22.63 \pm 4.77$ & 17.126 & $<0.001$ \\
\hline $\mathrm{t}$ & & 1.091 & 1.352 & & \\
\hline P-value & & 0.174 & 0.026 & & \\
\hline
\end{tabular}

Table IV. Comparison of the Barthel score between the two groups at 3 months after operation [n (\%)].

\begin{tabular}{lccccc}
\hline Group & $\begin{array}{c}\text { No. of } \\
\text { survived cases }\end{array}$ & $\begin{array}{c}\text { Self-care ability } \\
(100 \text { points })\end{array}$ & $\begin{array}{c}\text { Mild dysfunction } \\
(60-99 \text { points })\end{array}$ & $\begin{array}{c}\text { Moderate dysfunction } \\
(41-60 \text { points })\end{array}$ & $\begin{array}{c}\text { Severe dysfunction } \\
(\leq 40 \text { points })\end{array}$ \\
\hline Minimally invasion group & 34 & $6(17.65)$ & $22(64.71)$ & $5(14.71)$ & $1(2.94)$ \\
Craniotomy group & 35 & $7(20.00)$ & $21(60.00)$ & $5(14.29)$ & $2(5.71)$ \\
$\chi^{2}$ & & 0.541 & 0.762 & 0.994 & 0.805 \\
P-value & & 0.372 & 0.248 & 0.116 & 0.129 \\
\hline
\end{tabular}

\section{Results}

Comparison of postoperative complications. After 3 months of follow-up, there were no significant differences in the incidence rate of postoperative complications such as rehemorrhage or new intracranial hemorrhage and mortality rate (p>0.05) (Table II).

Comparison of the CSS score. Before operation, there was no significant difference in the CSS score between the two groups $(\mathrm{p}>0.05)$. At 28 days after operation, the CSS score in the minimally invasive group $(20.04 \pm 3.51$ points) was lower than that in the craniotomy group $(22.63 \pm 4.77$ points $)$ $(\mathrm{p}<0.05)$ (Table III).

Comparison of the Barthel score. At 3 months after operation, there were no significant differences in the scores of self-care ability, mild dysfunction, moderate dysfunction and severe dysfunction between the two groups ( $\mathrm{p}>0.05$ ) (Table IV).

Comparison of serum S-100 $\beta$. Through treatment, the levels of serum S-100 $\beta$ in both groups displayed a gradual decreasing

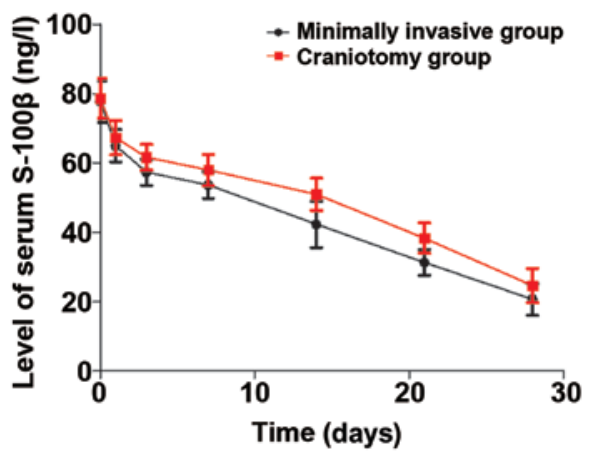

Figure 1. Changes in the content of serum S-100 $\beta$ in the two groups.

trend. The level of S-100 $\beta$ in the minimally invasive group was remarkably lower than that in the craniotomy group at 28 days after operation $(\mathrm{p}<0.05)$ (Fig. 1).

Comparisons of various MEP indexes. At 1 week after operation, 35 patients in the minimally invasive group were able to elicit MEP waveforms, and only 7 patients in the craniotomy group were able to elicit positive waveforms. At 2 weeks after 
Table V. Comparison of various MEP indexes between the two groups at 2 weeks after operation.

\begin{tabular}{lcccc}
\hline Group & $\begin{array}{c}\text { No. of } \\
\text { survived cases }\end{array}$ & $\begin{array}{c}\text { Incubation } \\
\text { period (msec) }\end{array}$ & $\begin{array}{c}\text { Central motor } \\
\text { conduction time (msec) }\end{array}$ & Amplitude (mV) \\
\hline Minimally invasion group & 40 & $21.74 \pm 2.68$ & $8.78 \pm 1.35$ & $9.68 \pm 5.44$ \\
Craniotomy group & 40 & $42.15 \pm 3.27$ & $13.76 \pm 1.92$ & $3.42 \pm 1.75$ \\
t & & 6.941 & 5.432 & 6.017 \\
P-value & 0.024 & 0.033 & 0.021 \\
\hline
\end{tabular}

MEP, motor-evoked potential.

Table VI. Comparison of operation-related indexes between the two groups (mean \pm SD).

\begin{tabular}{lccccc}
\hline Group & $\begin{array}{c}\text { No. of } \\
\text { survived cases }\end{array}$ & $\begin{array}{c}\text { Operation } \\
\text { time (min) }\end{array}$ & $\begin{array}{c}\text { Drainage tube } \\
\text { removal time (days) }\end{array}$ & $\begin{array}{c}\text { Length of hospital } \\
\text { stay (days) }\end{array}$ & $\begin{array}{c}\text { Total expenses } \\
\text { of hospitalization } \\
\text { (RMB } ¥ 0^{4} \text { ) }\end{array}$ \\
\hline Minimally invasion group & 34 & $71.69 \pm 15.42$ & $5.65 \pm 1.64$ & $20.57 \pm 4.32$ & $17.96 \pm 2.89$ \\
Craniotomy group & 35 & $104.85 \pm 26.44$ & $4.91 \pm 1.53$ & $25.34 \pm 5.45$ & $12.55 \pm 1.95$ \\
$\mathrm{t}$ & & 7.276 & 1.341 & 8.125 & 7.954 \\
P-value & 0.017 & 0.088 & 0.012 & 0.029 \\
\hline
\end{tabular}

operation, 40 patients in the minimally invasive and 20 patients in the craniotomy group could elicit MEP waveforms. The incubation period, central motor conduction time, and amplitude in the former were significantly better than those in the latter $(\mathrm{p}<0.05)($ Table V).

Comparison of operation-related indexes. There was no significant difference in the drainage tube removal time between the two groups $(p>0.05)$. The operation time and length of hospital stay were shorter with more total expenses of hospitalization in the minimally invasive group compared to those in the craniotomy group $(\mathrm{p}<0.05)$ (Table VI).

\section{Discussion}

The efficacy of conservative treatments for hypertensive intracerebral hemorrhage is currently not ideal, and surgical treatment is an important method to treat this kind of disease. Relevant data have indicated $(11,12)$ that the application of traditional craniotomy evacuation of hematomas is limited in clinical practice due to iatrogenic injury. Small bone window craniotomy is an improved technique based on traditional craniotomy, which is currently applied in the treatment of intracranial superficial hematomas. However, hematomas in the deep site increase the damage to brain tissues and reduce the significance of cerebral hemorrhage treatment $(13,14)$. In recent years, as the understanding of the minimally invasive techniques continues to deepen, the application of minimally invasive puncture and drainage for hematomas in the treatment of cerebral hemorrhage has gradually drawn attention (15). The principle of minimally invasive puncture and drainage for hematomas is to pierce a puncture needle into the patient's hematoma lesion via an electric drill to construct a channel for hematoma evacuation. Subsequently, the biochemical enzyme technique is adopted to liquefy and extract hematomas, thus achieving hematoma evacuation $(16,17)$. The results of the present study manifested that at 28 days after operation, the CSS score was $20.04 \pm 3.51$ points in the minimally invasive group, which was lower than that in the craniotomy group $(22.63 \pm 4.77$ points $)(\mathrm{p}<0.05)$, in consistency with other research findings (18).

After 3 months of follow-up, there were no significant differences in the incidence rate of postoperative complications such as rehemorrhage or new intracranial hemorrhage, the mortality rate, and the Barthel score $(p>0.05)$, suggesting that the efficacies of the two treatment options were identical in the aspects of complications, mortality rate and the ability to daily living activities. The operation time and length of hospital stay were shorter with more total expenses of hospitalization in the minimally invasive group compared to those in the craniotomy group $(\mathrm{p}<0.05)$, which is in agreement with the literature (19) and may be related to factors such as the operation procedures and anesthetic methods of the above two operations.

According to relevant data (20), serum S-100 $\beta$ is involved in the occurrence and development of cerebral hemorrhage, and the prognosis of patients with cerebral hemorrhage can be evaluated by measuring the level of serum S-100 $\beta$. This study revealed that the level of S-100 $\beta$ in the minimally invasive group was notably lower than that in the craniotomy group at 28 days after operation $(\mathrm{p}<0.05)$, suggesting that the minimally invasive puncture and drainage for hematomas can reduce more effectively the expression of serum $\mathrm{S}-100 \beta$ than the small bone marrow craniotomy evacuation of hematomas. The reason may be that the minimally invasive puncture and drainage for hematomas does not aggravate brain and systemic 
injuries in patients. The evacuation of hematomas can reduce the mechanical compression of them in patients, and the inflammatory damage mediated by them can be decreased through reducing the content of serum S-100 $\beta$.

Relevant data have manifested (21) that MEPs can be used to evaluate motor function (pyramidal beam function). Clinically, MEP is generally employed for the recovery of motor function after stroke. The results of this study demonstrated that at 1 week after operation, 35 patients in the minimally invasive group were able to elicit MEP waveforms, and only 7 patients in the craniotomy group were able to elicit positive waveforms. At 2 weeks after surgery, 40 patients in the minimally invasive group could elicit MEP waveforms, and 20 patients in the craniotomy group could elicit MEP waveforms. The incubation period, central motor conduction time and amplitude in the minimally invasive group were significantly better than those in the craniotomy group $(\mathrm{p}<0.05)$. In the craniotomy group, 20 patients were not able to elicit positive MEP waveforms at 2 weeks postoperatively, suggesting that the patient's motor center cannot produce nerve impulses at this time. It is worth mentioning that MEP can indicate the neurological function. Besides, the lack and absence of MEPs suggest that the neurological function may not be well restored (22). The above evaluation results of MEPs further suggest that the minimally invasive puncture and drainage for the clinical treatment of hypertensive cerebral hemorrhage has better clinical efficacy.

In summary, compared with the small bone window craniotomy, the minimally invasive puncture reduces the content of serum S-100 $\beta$. Its advantages are obvious, so it is worthy of promotion and application.

\section{Acknowledgements}

Not applicable.

\section{Funding}

No funding was received.

\section{Availability of data and materials}

The datasets used and/or analyzed during the current study are available from the corresponding author on reasonable request.

\section{Authors' contributions}

LL wrote and revised the manuscript. LL, HS and ML treated the patients and were also involved in the conception of the study. LL and HS recorded and analyzed the operation-related indexes. GL interpreted the neurological function. WP was responsible for serum S-100 $\beta$ detection. All authors read and approved the final manuscript.

\section{Ethics approval and consent to participate}

This study was approved by the Ethics Committee of Chengyang People's Hospital (Qingdao, China). Patients who participated in this research had complete clinical data. Signed informed consents were obtained from the patients or the guardians.

\section{Patient consent for publication}

Not applicable.

\section{Competing interests}

The authors declare that they have no competing interests.

\section{References}

1. Chen G, Ping L, Zhou S, Liu W, Liu L, Zhang D, Li Z, Tian Y and Chen Z: Early prediction of death in acute hypertensive intracerebral hemorrhage. Exp Ther Med 11: 83-88, 2016.

2. Doden T, Sato H, Sasahara E, Murata T, Yako T, Kitazawa K, Higuchi K, Kobayashi S and Hashimoto T: Clinico-radiological characteristics and pathological diagnosis of cerebral amyloid angiopathy-related intracerebral hemorrhage. J Stroke Cerebrovasc Dis 25: 1736-1745, 2016.

3. Fouda AY, Artham S, El-Remessy AB and Fagan SC: Renin-angiotensin system as a potential therapeutic target in stroke and retinopathy: Experimental and clinical evidence. Clin Sci (Lond) 130: 221-238, 2016.

4. Hu R and Feng H: Lenticulostriate artery and lenticulostriate-artery neural complex: New concept for intracerebral hemorrhage. Curr Pharm Des 23: 2206-2211, 2017.

5. Matsumoto K, Sakaki S, Abekura M and Yoshimine T: Co-existence of unruptured cerebral aneurysms in patients with hypertensive intracerebral hemorrhage. Acta Neurochir (Wien) 146: 1085-1089, discussion 1089, 2004.

6. Tian X, Shu H, Zhang H, Wang $\mathrm{H}$ and Guo L: Intracranial hemorrhage due to rupture of an anterior communicating artery aneurysm in a patient with pituitary adenoma. J Craniofac Surg 26: e154-e155, 2015.

7. Yiannakopoulos CK: Carpal ligament decompression under local anaesthesia: The effect of lidocaine warming and alkalinisation on infiltration pain. J Hand Surg Br 29: 32-34, 2004.

8. Zhang Y, Liu B, Liu Z, Wang Y, Zhao H, Zi M, Wang L, Liu H, Chen Z and Xie Y: Development of a disease-specific health-related quality of life questionnaire for patients with post-stroke spasticity. J Tradit Chin Med 32: 674-678, 2012.

9. Lue YJ, Lin RF, Chen SS and Lu YM: Measurement of the functional status of patients with different types of muscular dystrophy. Kaohsiung J Med Sci 25: 325-333, 2009.

10. Kenning TJ, Dalfino JC, German JW, Drazin D and Adamo MA: Analysis of the subdural evacuating port system for the treatment of subacute and chronic subdural hematomas. J Neurosurg 113: 1004-1010, 2010.

11. Southwell DG, Hervey-Jumper SL, Perry DW and Berger MS: Intraoperative mapping during repeat awake craniotomy reveals the functional plasticity of adult cortex. J Neurosurg 124: $1460-1469,2016$

12. Velnar T and Bunc G: Iatrogenic metastasis of a benign meningioma to the periosteum at the site of previous craniotomy: A case report. Wien Klin Wochenschr 120: 766-769, 2008.

13. Chi FL, Lang TC, Sun SJ, Tang XJ, Xu SY, Zheng HB and Zhao HS: Relationship between different surgical methods, hemorrhage position, hemorrhage volume, surgical timing, and treatment outcome of hypertensive intracerebral hemorrhage. World J Emerg Med 5: 203-208, 2014.

14. Morotti A, Paciaroni M, Zini A, Silvestrelli G, Del Zotto E, Caso V, Dell'Acqua ML, Simone AM, Lanari A, Costa P, et al: Risk profile of symptomatic lacunar stroke versus nonlobar intracerebral hemorrhage. Stroke 47: 2141-2143, 2016.

15. Han WY, Tao YQ, Xu F, Zhang YQ, Li ZY and Liang GB: The short- and long-term efficacy analysis of stereotactic surgery combined external ventricular drainage in the treatment of the secondary intraventricular hemorrhage. Brain Behav 7: e00864, 2017.

16. Guldberg-Kjär T and Johansson B: ADHD symptoms across the lifespan: A comparison of symptoms captured by the Wender and Barkley Scales and DSM-IV criteria in a population-based Swedish sample aged 65 to 80. J Atten Disord 19: 390-404, 2015. 
17. Sonni S, Lioutas VA and Selim MH: New avenues for treatment of intracranial hemorrhage. Curr Treat Options Cardiovase Med 16: 277, 2014

18. Wang WZ, Jiang B, Liu HM,Li D,Lu CZ,Zhao YD and Sander JW: Minimally invasive craniopuncture therapy vs. conservative treatment for spontaneous intracerebral hemorrhage: Results from a randomized clinical trial in China. Int J Stroke 4: 11-16, 2009.

19. James ML, Blessing R, Phillips-Bute BG, Bennett E and Laskowitz DT: S100B and brain natriuretic peptide predict functional neurological outcome after intracerebral haemorrhage. Biomarkers 14: 388-394, 2009.

20. Ikedo T, Nakamura K, Sano N, Nagata M, Okada Y, Terakawa Y and Murata T: Intraoperative transcranial motor-evoked potentials predict motor function outcome in intracerebral hemorrhage surgery. World Neurosurg 90: 518-523, 2016.
21. Voulgaris S, Karagiorgiadis D, Alexiou GA, Mihos E, Zigouris A, Fotakopoulos G, Drosos D and Pahaturidis D: Continuous intraoperative electromyographic and transcranial motor evoked potential recordings in spinal stenosis surgery. J Clin Neurosci 17: 274-276, 2010.

22. Bestmann S and Krakauer JW: The uses and interpretations of the motor-evoked potential for understanding behaviour. Exp Brain Res 233: 679-689, 2015.

(i) (9) This work is licensed under a Creative Commons Attribution-NonCommercial-NoDerivatives 4.0 International (CC BY-NC-ND 4.0) License. 